\title{
New molecularly-imprinted polymer for carnitine and its application as ionophore in potentiometric selective membranes
}

\author{
Joséphine Moret, Felismina T.C. Moreira, Sofia A.A. Almeida , M. Goreti F. Sales
}

\section{A B S T R A C T}

Carnitine (CRT) is a biological metabolite found in urine that contributes in assessingseveral disease conditions, including cancer. Novel quick screening procedures for CRT are therefore fundamental. This work proposes a novel potentiometric device where molecularly imprinted polymers (MIPs) were used as ionophores. The host-tailored sites were imprinted on a polymeric network assembled by radical polymerization of methacrylic acid (MAA) and trimethylpropane trimethacrylate (TRIM). Non-imprinted polymers (NIPs) were produced as control by removing the template from the reaction media. The selective membrane was prepared by dispersing MIP or NIP particles in plasticizer and poly(vinyl chloride), PVC, and casting this mixture over a solid contact support made of graphite.

The composition of the selective membrane was investigated with regard to kind/amount of sensory material (MIP or NIP), and the need for a lipophilic additive. Overall, MIP sensors with additive exhibited the best performance, with near-Nernstian response down to $\sim 1 \times 10^{-4} \mathrm{~mol} \mathrm{~L}^{-1}$, at $\mathrm{pH} 5$, and a detection limitof $\sim 8 \times$ $10^{-5} \mathrm{~mol} \mathrm{~L}^{-1}$. Suitable selectivity was found for all membranes, assessed by the matched potential method against some of the most common species in urine (urea, sodium, creatinine, sulfate, fructose and hemoglobin). CRT selective membranes including MIP materials were applied successfully to the potentiometric determination of CRT in urine samples.

\section{Keywords:}

Carnitine

Molecularly-imprinted polymer

Sensor

Potentiometry

Urine

\section{Introduction}

Cancer diseases are responsible for about $20 \%$ of deaths in the European Region, most of these are attributed to breast, cervical or colorectal cancer $[1,2]$. Screening programs and early diagnostic will be more effective once they rely on non- or minimally-invasive methods with high sensitivity and specificity, easily available and cost effective. These may be reached by the early detection of biomarker proteins that are found early in the body and that have predictive value for specific diseases.

Carnitine (CRT) is a quaternary ammonium compound biosynthesized from the amino acids lysine and methionine and is responsible in living cells for the transport of long-chain fatty acids into mitochondria where $\beta$-oxidation takes place [3]. CRT is a potential biomarker of ovarian cancer, it was identified as a metabolite in the normal ovary and transformed in primary and metastatic ovarian cancer [4,5]. It is however widely recognized by its antioxidant properties, by which it may become an efficient tool at the prevention and protection of oxidative stress related diseases, including heart failure [6], lipid peroxidation [7], Down syndrome [8], aging [9] and HIV [10]. Furthermore, CRT has also correlated to the inhibition of carcinogenesis and cisplatin-induced injury of kidney and intestine [11,12], and to antidiabetic activity since the long-chain free fatty acid transport by CRT is associated withthe glucose metabolism $[13,14]$. There is also a close correlation between L-CRT supplementation and the beneficial effects in the treatment of varicocele, a major cause of male infertility [15].

Several analytical methods have been reported in the literature to analyze CRT in pharmaceutical formulations and in biological samples such as chromatographic techniques [16-19], capillary zone electrophoresis [20-22], fluorimetry[23], spectrophotometry[24], and electrochemical assays with potentiometric [25-27], amperometric [28] or quartz-crystal microbalance transduction [29]. Typically, separative methods, such as chromatographic and capillary zone electrophoresis, are accurate, precise and robust, but are unsuitable for routine applications for requiring high sample running times, organic solvents, pretreating sample procedures, expensive equipment, and high purity requirements for reagents. Optical methods such as fluorimetry and spectrophotometry are typically much less expensive but may lack the desired selectivity and the ability to handle easily turbid and colored samples. Other methods reported in the literature include potentiometric titrations, as an indirect measure of CRT that is not suitable for the direct analysis of complex samples. Other methods make use of antibody/ 
antigen reactions [28,29], providing specific and sensitive responses but requiring long time and expensive reagents for routine analytical readings.

Ion selective electrodes (ISEs) may turn out an advantageous alternative for CRT determination in complex samples. CRT bears a positive charge from a quaternary ammonium group and has one carboxylic acid function that may lead to one negative charge, meaning that its detection as an ionic species is feasible. The use of ISEs also provides a highly specific and sensitive measurement and offers high precision and rapidity, low cost of analysis, and enhanced selectivity and sensitivity over a wide range of concentrations [30-32]. In this, an ionophore is immobilized in a plasticized membrane, acting as an electroactive material.

Conventional ionophores are ion exchangers and neutral macrocyclic compounds [25-27], but it has been shown that new materials that are complementary to the size and charge of a particular ion can lead to very selective interactions, thus enhancing the selectivity of the final device. This is the case of molecularly imprinted polymers (MIPs), by which polymeric materials are easily tailored with suitable selectivity for a guest compound [33,34]. There are various successful applications of MIPs, including chromatography [35,36], artificial antibodies [37-39], chemical sensors [40,41], and solid-phase extraction (SPE) $[42,43]$, as well as potentiometric ionophores[44,45].

The present work describes the development of CRT MIP-based ISEs. The imprinted material is synthesized by cross-linking the polymerization of methacrylic acid (MAA) with trimethylolpropane trimethacrylate (TRIM) within the template molecule. The sensing materials are dispersed in a PVC matrix plasticized with o-nitrophenyl octyl ether (oNPOE). Main analytical features, selectivity properties and practical application to urine analysis are presented.

\section{Experimental}

\subsection{Apparatus and chemicals}

Practical potentials were measured in a pH meter, Crison, GLP 21 ( $\pm 0.1 \mathrm{mV}$ sensitivity), at room temperature, and under constant stirring. A home-made commutation unit with one six-way out and one-way in was employed to enable the simultaneous reading of six ISEs. The reference electrode was $\mathrm{Ag} / \mathrm{AgCl}$, of double junction from Crison, 52-40. The selective electrode was constructed as described elsewhere [46], holding conventional configuration, no internal reference solution and an epoxy-graphite conductive support. The potentiometric cell was assembled as: conductive graphite | CRT selective membrane | buffered sample solution (MES, $1.0 \times 10^{-2} \mathrm{~mol} \mathrm{~L}^{-1}$, pH 5) || electrolyte solution, $\mathrm{KCl}|\mathrm{AgCl}(\mathrm{s})| \mathrm{Ag}$. All pH measurements used a combined glass electrode from Crison.

\subsection{Reagents}

All chemicals were of analytical grade and de-ionized water (conductivity $<0.1 \mu \mathrm{cm}^{-1}$ ) was employed throughout. CRT, potassium tetrakis(4-chlorophenyl)borate (TpClTPB), oNPOE, 4-(2hydroxyethyl)-1-piperazineethanesulfonic acid (HEPES), poly(vinyl chloride) (PVC) of high molecular weight, trimethylpropane trimethacrylate (TRIM), hemoglobin and methacrylic acid (MAA) were purchased from Fluka. Benzoyl peroxide (BPO), acetonitrile $(\mathrm{ACN})$, magnesium chloride and tetrahydrofuran (THF) were from Riedel-deHäen, urea from Fagron, fructose and sodium chloride from Pancreac and sodium sulfate from Normapur.

\subsection{Synthesis of imprinted-ionophore}

MIP materials were obtained by dissolving the template (CRT, $1.0 \mathrm{mmol}$ ) with the functional monomer (5.0 mmol, MAA), the crosslinker (TRIM, $20.0 \mathrm{mmol}$ ) and the radical initiator (BPO, $0.32 \mathrm{mmol}$ ), in $3.5 \mathrm{~mL}$ acetonitrile. The mixture was sonicated, degassed with nitrogen for $10 \mathrm{~min}$, and cured at $70{ }^{\circ} \mathrm{C}$ for $1 \mathrm{~h}$ and $30 \mathrm{~min}$. Non-imprinted polymers (NIPs) were prepared in parallel, repeating this procedure without the template. The resulting polymers were then crushed dried to small-size particles. Template, exceeding reactants and small size by-products were extracted from the particles by thorough washing with acetonitrile/acetic acid (5:1, v/v). The absence of CRT within the acetonitrile/acetic acid MIP material washing extracts was confirmed by UV/Vis spectrophotometry. Finally, the particles were washed with water and dried, at room temperature, for $12 \mathrm{~h}$, and kept in a dessicator.

\subsection{Surface analysis of the host-tailored polymers}

FTIR analysis was conducted in a Nicolet 6700 FTIR spectrometer (Thermo) coupled to a diamond ATR accessory of the same brand. Infrared spectra were collected under room temperature control after background correction. The number of scans was 32 for both sample and background. The collected spectra plotted wavenumber (525 to $4000 \mathrm{~cm}^{-1}$ ) against \% transmittance. Resolution was 4000 .

Raman microscopy was made in Raman microscope, equipped with $532 \mathrm{~nm}$ laser. The spectrum was measured using $50 \mu \mathrm{m}$ pinhole aperture and $50 \times$ lens.

\subsection{Preparation of CRT-selective electrodes}

The sensing membranes were prepared by mixing $0.21 \mathrm{~g}$ of PVC, 0.35 $\mathrm{g}$ of plasticizer $\mathrm{oNPOE}$ and $0.015 \mathrm{~g}$ or $0.00375 \mathrm{~g}$ of the sensing polymer (Table 1) in $3.5 \mathrm{~mL}$ THF. Some membranes also included $0.007 \mathrm{~g}$ or $0.0007 \mathrm{~g}$ of TpClTPB, acting as lipophilic additive.

The resulting membrane solutions were drop-casted over the conductive support of the conventional electrodes. Membranes were let dry for $24 \mathrm{~h}$ and conditioned in water. The electrodes were kept in this solution when not in use.

\subsection{Potentiometric procedures}

All measurements were made at room temperature. Calibration curves were done in constant ionic strength and $\mathrm{pH}$ conditions, using $1.0 \times 10^{-2} \mathrm{~mol} \mathrm{~L}^{-1}$ HEPES buffer solution as background, adjusted to the desired $\mathrm{pH}$ with $\mathrm{NaOH}$ or $\mathrm{HCl}$ solution. The selective membrane of the electrodes was incubated in $50.00 \mathrm{~mL}$ buffer, where increasing aliquots of a $1.0 \times 10^{-2} \mathrm{~mol} \mathrm{~L}^{-1}$ stock solution of CRT $(0.0200-$ $5.00 \mathrm{~mL}$ ) were added. Emf readings were recorded after stabilization to $\pm 0.2 \mathrm{mV}$ and the values was plotted as a function of logarithm CRT concentration.

Potentiometric selectivity coefficients were assessed by the matched potential method (MPM). For this purpose, a known amount of the primary ion $(a A)$ in solution was added of a known amount of primary ion $\left(a A^{\prime}\right)$, and the corresponding potential change $(\Delta \mathrm{E})$ is recorded. Then, another solution of the same amount of primary ion $(a A)$ is added of interfering ion $(a B)$ until the same potential change $(\Delta E)$ is met.

$K_{A, B}^{P O T}=\frac{(a A \ddot{\mathbf{E}}-a A)}{a B}$.

The initial concentration of primary ion was set to $9.9 \times 10^{-5} \mathrm{~mol} / \mathrm{L}$ $(a A)$ and the concentration of primary ion solution added into this was $4.8 \times 10^{-4} \mathrm{~mol} \mathrm{~L}^{-1}\left(a A^{\prime}\right)$. The corresponding potential change depended on the composition of the selective membrane, being $\sim 20 \mathrm{mV}$ on MIP/additive devices. The interference of creatinine, urea, sodium, potassium, calcium and sulfate was assessed by adding small aliquots of the corresponding solutions into the primary ion solution of $a A$. This addition was made consecutively until one of the following conditions was met: the potential change equaled the one observed with the main ion addition for the same electrode; or the initial concentration of primary ion was altered by more than $5 \%$ as aresult of dilution. 
Table 1

Membrane composition of PVC CNT selective membranes and their mean potentiometric features $(n=3)$ in $1.0 \times 10^{-2}$ mol/L HEPES buffer, pH 5 .

\begin{tabular}{|c|c|c|c|c|c|c|c|c|}
\hline \multirow[t]{2}{*}{ ISE } & \multicolumn{3}{|c|}{ Membrane composition } & \multirow{2}{*}{$\begin{array}{l}\text { Slope } \\
\left(\mathrm{mV} \text { decade }^{-1}\right)\end{array}$} & \multirow{2}{*}{$\begin{array}{l}\mathrm{R}^{2} \\
(n=3)\end{array}$} & \multirow{2}{*}{$\begin{array}{l}\text { LOD } \\
(\mathrm{mol} / \mathrm{L})\end{array}$} & \multirow{2}{*}{$\begin{array}{l}\text { LLLR } \\
(\mathrm{mol} / \mathrm{L})\end{array}$} & \multirow{2}{*}{$\begin{array}{l}\text { ov } \\
(\mathrm{mV})\end{array}$} \\
\hline & Ionophore & Plasticizer & Additive & & & & & \\
\hline I & MIP (15 mg) & oNFOE (350 mg) & - & $42.98 \pm 2.92$ & 0.9921 & $5.77 \times 10^{-5}$ & $9.07 \times 10^{-5}$ & 1.80 \\
\hline II & MIP (15 mg) & oNFOE (350 mg) & TpCIPB (7.0 mg) & $51.43 \pm 2.35$ & 0.9947 & $9.96 \times 10^{-5}$ & $1.53 \times 10^{-4}$ & 1.10 \\
\hline III & NIP (15 mg) & oNFOE (350 mg) & - & $38.06 \pm 2.67$ & 0.9968 & $7.74 \times 10^{-5}$ & $9.07 \times 10^{-5}$ & 3.56 \\
\hline IV & NIP (15 mg) & oNFOE (350 mg) & TpClPB (7.0 mg) & $63.73 \pm 2.23$ & 0.9912 & $1.89 \times 10^{-4}$ & $1.99 \times 10^{-4}$ & 2.42 \\
\hline V & MIP (3.8 mg) & oNFOE (350 mg) & - & $20.12 \pm 1.31$ & 0.9859 & $5.24 \times 10^{-5}$ & $9.30 \times 10^{-5}$ & 1.70 \\
\hline VI & MIP (3.8 mg) & oNFOE (350 mg) & TpCIPB (0.7 mg) & $49.47 \pm 2.42$ & 0.9945 & $8.15 \times 10^{-5}$ & $1.14 \times 10^{-4}$ & 0.76 \\
\hline VII & NIP (3.8 mg) & oNFOE (350 mg) & TpCIPB (0.7 mg) & $62.75 \pm 16.45$ & 0.9942 & $1.49 \times 10^{-4}$ & $2.39 \times 10^{-4}$ & 13.99 \\
\hline VIII & - & oNFOE (350 mg) & TpClPB (7.0 mg) & $70.45 \pm 7.98$ & 0.9919 & $1.68 \times 10^{-4}$ & $2.38 \times 10^{-4}$ & 16.04 \\
\hline IX & - & oNFOE (350 mg) & TpCIPB (0.7 mg) & $82.38 \pm 14.82$ & 0.9870 & $2.32 \times 10^{-4}$ & $3.64 \times 10^{-4}$ & 3.49 \\
\hline $\mathrm{X}$ & - & oNFOE (350 mg) & - & $26.14 \pm 1.99$ & 0.9961 & $7.48 \times 10^{-5}$ & $7.73 \times 10^{-5}$ & 4.57 \\
\hline
\end{tabular}

Considering that urine samples would be diluted prior to analysis, all interfering species were tested up to half of their typical concentrations in urine $(0.33$ urea $\mathrm{mol} / \mathrm{L}, 0.0088 \mathrm{~mol} / \mathrm{L}$ creatinine, 0.26 sodium mol/L; 0.038 potassium $\mathrm{mol} / \mathrm{L} ; 0.0037$ calcium $\mathrm{mol} / \mathrm{L} ; 0.0041$ magnesium $\mathrm{mol} / \mathrm{L} ; 0.017$ chloride $\mathrm{mol} / \mathrm{L} ; 0.038$ sulfate $\mathrm{mol} / \mathrm{L} ; 0.0014$ fructose $\mathrm{mol} / \mathrm{L}$; and $4.41 \times 10^{-8} \mathrm{~mol} / \mathrm{L}$ hemoglobin).

For the analysis of biological fluids, urine and serum were diluted with $1.0 \times 10^{-2} \mathrm{~mol} / \mathrm{L}$ HEPES buffer $(\mathrm{pH} 5)$. The direct method was applied to determine CRT in these samples. The samples were spiked with 165.7 and $300.1 \mathrm{mg} / \mathrm{L}$ ofCRT.

\subsection{Segmented sandwich membrane method}

The segmented sandwich membrane method was used [47]. In this, two layers of selective membrane were casted over the conductive support, the first one considered as blank for missing the active ingredient and the second one including the active ingredient and contacting directly with the measuring solution. Conventionally, the first membrane should be a blank to the membrane under study. So, two kinds of blank materials were tested: either without ionophore (MIP/NIP) or without both ionophore and additive. So, four different electrodes including different membrane combinations were tested: (1) the second membrane was type VI (15 mg, MIP and $0.007 \mathrm{mg}$, TpCITPB, Table 1) the first one was the same but without MIP; (2) the second membrane was also type VI and the first one was the same but without MIP and additive; (3) the second membrane was type VII (15 mg, NIP and $0.007 \mathrm{mg}$, TpCITPB, Table 1) and the first one was the same but without NIP; and (4) the second membrane was also type VII and the first one was the same but without NIP and additive. The resulting electrodes were incubated in CRT standard solution, prepared in HEPES buffer, pH 5, and the emf measured in each 3 min until full stabilization.

\section{Results and discussions}

\subsection{CRT selective material}

CRT selective sensors were synthesized by means of molecularlyimprinted technique, as described in Fig. 1. This was done by placing the template (CRT) in an aprotic and a non-polar solvent, where the functional monomer (MAA) and cross-linking monomers (TRIM) were polymerized by the addition of BPO, a source of radical species. CRT species entrapped inside the polymeric matrix were removed by thorough washing. The exit of CRT created binding positions to which other CRT species could rebind later.

The interaction between CRT and the polymeric matrix was of noncovalent nature, expectedly ionic and hydrogen bonding. MAA has carboxylic acid function that acts as hydrogen acceptor/donor at the same time and may display a negative charge if in suitable $\mathrm{pH}$ conditions. CRT has a quaternary amine cation function that may establish ionic interactions with carboxylate group (negatively charged) and also carboxylic and hydroxyl functions that are hydrogen acceptor/donors. Overall, these non-covalent interactions may be reversed, typically by wash in aqueous solution of an acid, a base, or methanol [48].

\subsection{FTIR analysis of the synthetic polymers}

ATR-FTIR spectra of the prepared materials confirmed equivalent polymerization process in MIP and NIP particles as well as the absence of CRT on the MIP particles (Fig. 2).

Overall, MIP and NIP materials displayed similar FTIR spectra because they shared similar chemical functions (see chemical structure of monomer and cross linker in Fig. 1) once CRT was removed from the MIP polymeric matrix. Strong absorption peaks were found at about $1720 \mathrm{~cm}^{-1}$ and $1140 \mathrm{~cm}^{-1}$, corresponding to $\mathrm{C}=0$ or $\mathrm{C}-\mathrm{O}$ stretches, respectively. The peak at about $2950 \mathrm{~cm}^{-1}$ is assigned to $\mathrm{C}-\mathrm{H}$ stretching vibrations.

The absence of CRT in the MIP structure was evidenced by the absence of the absorption peak at $3389 \mathrm{~cm}^{-1}$, which could be correlated to non-bonded $\mathrm{O}-\mathrm{H}$ stretch band and/or $\mathrm{N}-\mathrm{C}$ ammonium bond stretch.

\subsection{General analytical performance of ISEs}

The CRT selective membrane sensors contained MIP or NIP particles as electroactive ingredients dispersed in PVC plasticized with oNPOE. Different selective membrane compositions were prepared, in order to assess the influence of the kind (MIP/NIP) and amount of ionophore and the need for a lipophilic additive (Table 1).

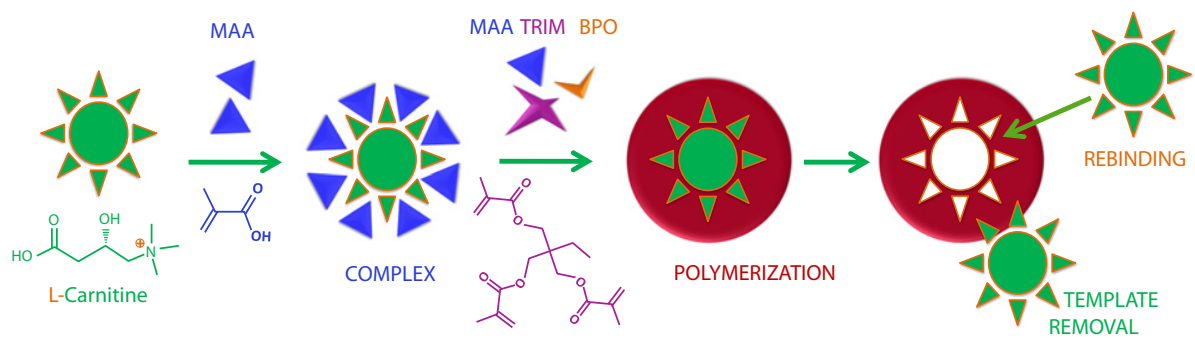

Fig. 1. Overall schematic representation of the imprinting process and the chemical structures of monomer, template and cross-linker. 


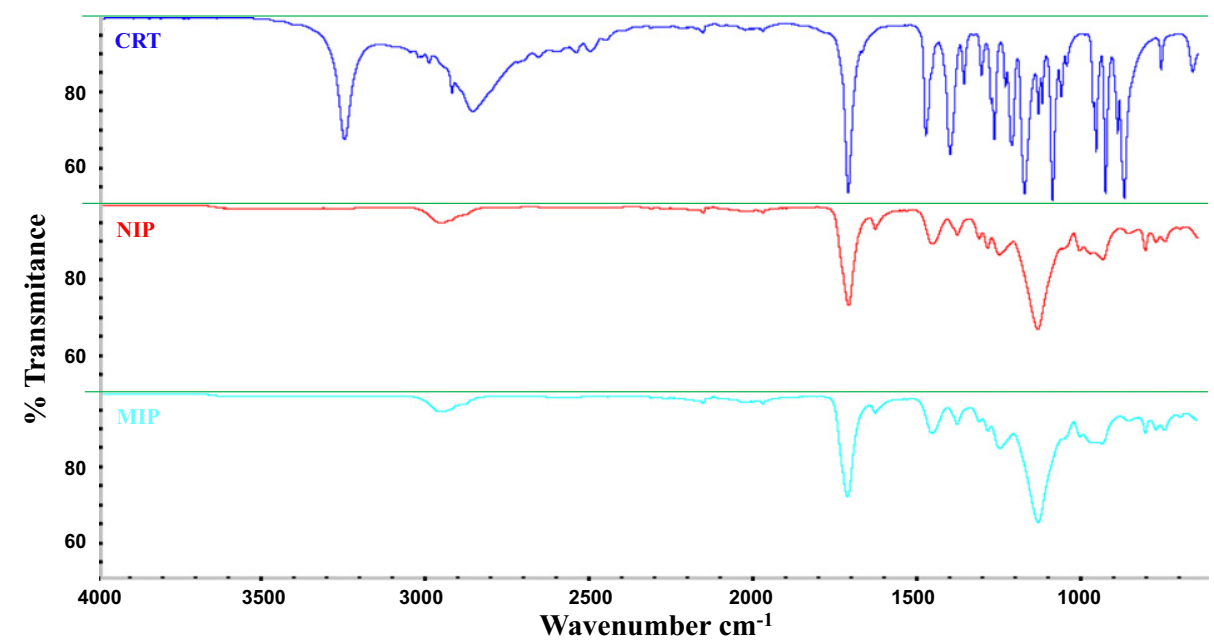

Fig. 2. FTIR spectra of the different sensory materials (MIP and NIP) and CRT as control.

The resulting membranes were observed by an optical microscope coupled to a Raman spectrometer, in order to understand the distribution of the particles inside the membrane. Overall, the particles seemed to have a homogenous distribution, with sizes $<2 \mu \mathrm{m}$ (Fig. 3, inset). The Raman spectrum of the material with or without PVC membrane was also given in the same figure, but no significant information may be extracted from it, apart from the fact that the coincident peaks between both spectra may probablybe correlated to the presence of the material inside the membrane.

The amount of electroactive material has a great impact on the sensitivity and the detection limits of the final device while the lipophilic additive may be necessary to ensure minimum ionic permselectivity leading to a potentiometric response (Fig. 4). The amount of electroactive material was set to $2.6 \%(\mathrm{w} / \mathrm{w}$, ISEs I to IV) and $0.7 \%(\mathrm{w} /$ $\mathrm{w}$, ISEs V to VII), using MIP (ISEs I, II, V and VI) or NIP (ISEs III, IV and VII) materials, and having additive in higher ( $7 \mathrm{mg}$, ISEs II and IV) or lower $(0.7 \mathrm{mg}$, ISEs VI and VII) amounts (in agreement with the amount of ionophore). Blank selective membranes were also prepared with plasticizer (ISE X) and additive (ISEs VIII and IX) in the same amounts previously indicated.

The main analytical features of all these ISEs were evaluated by IUPAC recommendations [49] and are shown in Table 1. Overall, the results pointed out that polymeric materials, both MIP and NIP, are able to act as ionophore for CRT, but the potentiometric response is clearly sub-Nernstian, a feature that is intensified for membranes with a lower amount of ionophore (Fig. 4, left). An improved response is achieved by adding a lipophilic additive of negative charge, thereby enhancing the detection capability for cationic species (Fig. 4, center). The magnification of the analytical response seemed more intense when NIP materials were used, but the potentiometric system became highly irreproducible, showed poorer linearity and was less sensitive than the MIP-based potentiometric systems. Blank membranes were able to establish a sensitive response for CRT only when $7 \mathrm{mg}$ of additive was present, which corresponded to $1.2 \%$ of this ionic additive in the membrane. This was already expected, but the reproducibility of the final electrode was not sufficient for any practical analytical application.

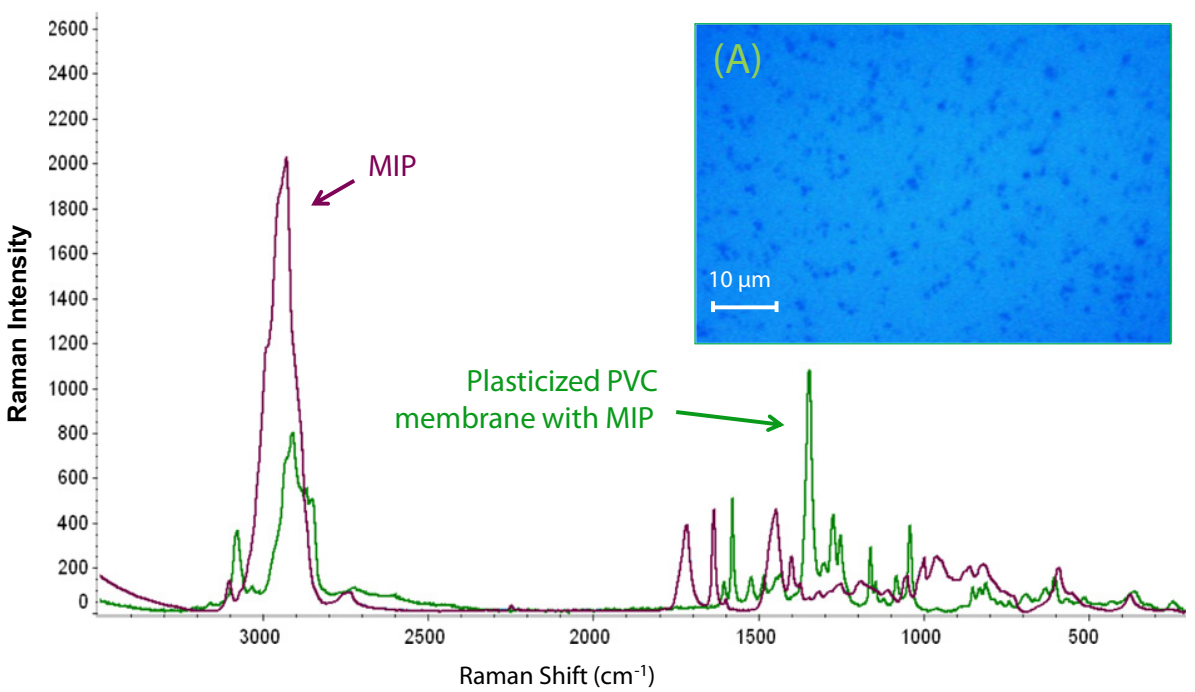

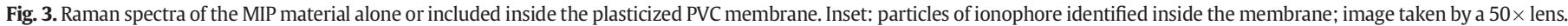




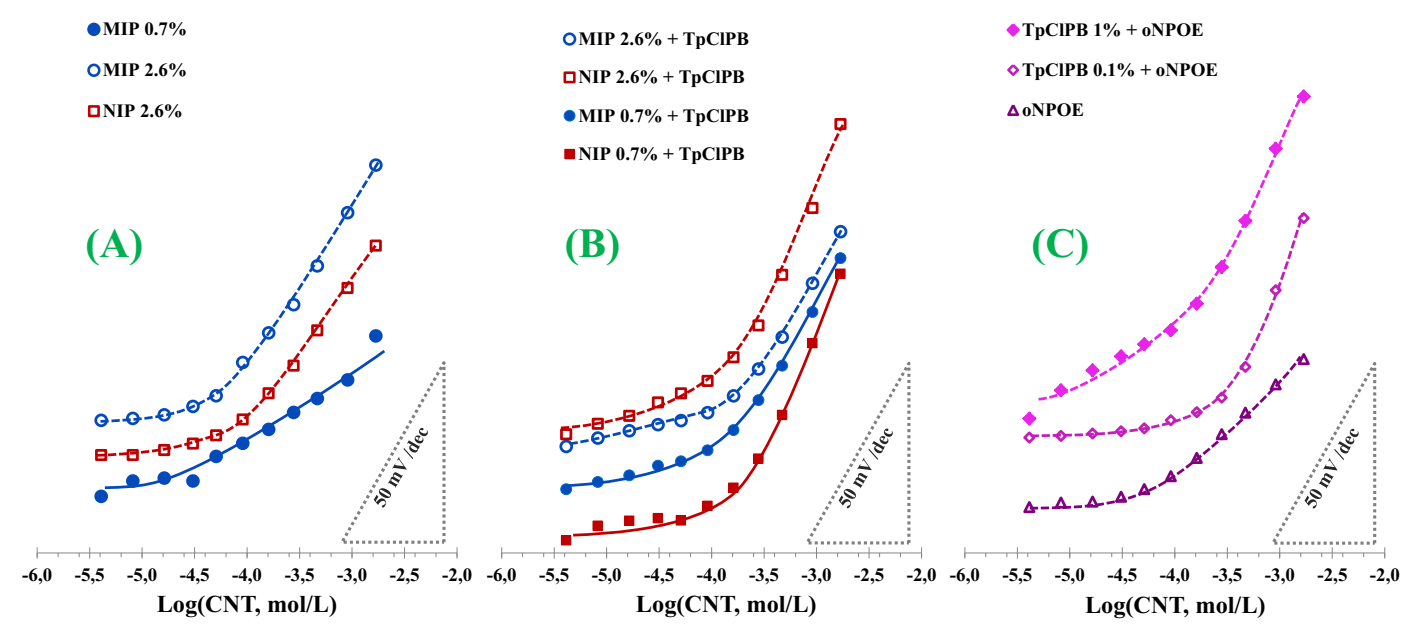

Fig. 4. Potentiometric response of CRT PVC membrane sensors according to their membrane composition (assays in pH 5).

Overall, ISEs II and VI displayed the most suitable combination of analytical features, presenting near-Nernstian cationic slopes, low detection and linear range limits and good reproducibility. The need for an additive was clear, but the amount by which it was added seemed irrelevant. MIP materials were also more suitable, especially in terms of reproducibility and detection capability.

\subsection{Selectivity of the sensors}

The selectivity of the ISEs was expressed in terms of potentiometric selectivity coefficients $\left(\mathrm{K}^{\mathrm{POT}}\right)$ that define the ability of an ISE to differentiate a primary ion from interfering ones. These $\mathrm{K}^{\mathrm{POT}}$ values were calculated by the MPM [50], where $\mathrm{K}^{\mathrm{POT}}$ corresponded to the ratio of primary (A) and interfering (B) ionconcentrations (activity in theory) that lead to the same potential change under identical conditions (Eq. (1)). Creatinine, fructose, sodium, urea, and sulfate, were tested as possible interfering species in biological samples. Overall, $\log \mathrm{K}^{\mathrm{POT}}$ ranged from -1.99 to -0.40 and the lowest value was obtained for sodium (Table 2). Many of the interfering species were unable to match the desired potential difference, meaning that their interference effect was negligible. This effect was most evident for fructose and hemoglobin.

With regard to the membrane composition, it seems clear that the presence of the additive enhanced the selectivity properties of the potentiometric device. Overall, combining selectivity and analytical features, the best selective membranes included MIP material and additive, regardless the quantity of these active ingredients.

\subsection{Binding of CRT-ionophore in the selective membrane}

The successful use of molecularly-imprinted materials as ionophore in selective membranes requires the formation of stable complexes between the imprinted template and their functional groups of the monomers on the polymeric matrix. In order to assess the extent of this complexation in the selective membrane environment, the stability constants between CRT and MIP/NIP were investigated by the approach of Mi and Bakker[47], following equation,

$\beta_{\mathrm{ILn}}=\left(\mathrm{L}_{\mathrm{T}}-\frac{n \mathrm{R}_{\mathrm{T}}}{\mathrm{z}_{i}}\right)^{-n} \exp \left(\frac{E_{M} Z_{I} F}{R T}\right)$

where $\mathrm{L}_{\mathrm{T}}$ is the total concentration of ionophore in the membrane segment; $\mathrm{R}_{\mathrm{T}}$ the concentration of lipophilic ionic site; $n$ is the ionionophore complex stoichiometry; and $\mathrm{R}, \mathrm{T}$ and $\mathrm{F}$ are the gas constant, the absolute temperature and the Faraday constant, respectively; $\mathrm{E}_{\mathrm{M}}$ the membrane potential; and the ion carries a charge of $z_{i}$. This method was adapted herein to the use of polymeric materials on solid contact electrodes.

The constant formation values of $\beta \mathrm{IL}_{n}$ obtained with membranes including imprinted material in ISE-1 and ISE-2 formats (please see Section 2.7) were similar, equal to 4.33 and 4.43 , respectively. The difference between these electrodes was the absence of additive in the membrane casted first, suggesting that the inclusion of additive as a blank did not affect the extent of complexation between CRT and the binding sites within the MIP material acting as ionophore. A similar result was observed for non-imprinted materials, tested as ISE-3 and ISE-4 formats and correlated to formation constants of -1.42 and -1.62 , respectively. A more extensive interpretation over this data is not possible because the absolute values obtained reflect the complex stability between ion and ionophore within the membrane phase but can only be compared within the electrodes presented herein.

Overall, the most important observation in this study was that MIPs displayed higher ability to complex with CRT in a plasticized membrane environment than NIP materials. This evidenced the need for imprinting the polymeric structure in order to generate emf values that are strongly

Table 2

Potentiometric selectivity coefficients of the CNT selective electrodes determined by the matched potential method in HEPES buffer, pH 5 .

\begin{tabular}{|c|c|c|c|c|c|c|c|c|c|c|}
\hline \multirow[t]{2}{*}{ Interfering species } & \multicolumn{10}{|c|}{$\log ^{\mathrm{POT}}$} \\
\hline & ISE I & ISE II & ISE III & ISE IV & ISE V & ISE VI & ISE VII & ISE VIII & ISE IX & ISE $X$ \\
\hline Creatinine & - & - & - & - & - & - & - & -1.37 & - & - \\
\hline Sodium & -1.64 & -1.64 & - & -1.22 & -1.22 & - & - & -1.99 & -1.64 & - \\
\hline Sulfate & -0.82 & - & -0.82 & -0.40 & -0.82 & - & - & - & -0.40 & -0.82 \\
\hline Urea & - & - & - & -0.55 & - & - & -1.09 & - & - & -1.67 \\
\hline Fructose & - & - & - & - & - & - & - & - & - & - \\
\hline Hemoglobin & - & - & - & - & - & - & - & - & - & - \\
\hline
\end{tabular}


Table 3

Potentiometric determination of CRT in spiked urine samples using electrodes with MIP material and additive.

\begin{tabular}{|c|c|c|c|c|c|}
\hline \multirow[t]{2}{*}{ Sample } & \multirow{2}{*}{$\begin{array}{l}\text { CRT } \\
\left(\mathrm{mg} \mathrm{L}^{-1}\right)\end{array}$} & \multicolumn{2}{|l|}{ ISE II } & \multicolumn{2}{|l|}{ ISE VI } \\
\hline & & Found ( $\left.\mathrm{mg} \mathrm{L}^{-1}\right)$ & Relative error \% & Found $\left(\mathrm{mg} \mathrm{L}^{-1}\right)$ & Relative error \% \\
\hline 1 & 135.12 & $137.87 \pm 2.52$ & $+2.04 \pm 1.87$ & $124.89 \pm 2.92$ & $-7.57 \pm 2.16$ \\
\hline 2 & 309.97 & $295.77 \pm 4.68$ & $-4.58 \pm 1.51$ & $279.94 \pm 4.69$ & $-9.69 \pm 1.51$ \\
\hline 3 & 614.0 & $557.0 \pm 4.0$ & $+9.16 \pm 1.51$ & $573 \pm 3.15$ & $+6.67 \pm 1.51$ \\
\hline
\end{tabular}

correlated to the complexation with the target ion and its imprinted binding site.

\subsection{Analytical application}

Urine samples were spiked with concentrations of 135 and $310 \mu \mathrm{g} /$ $\mathrm{mL}$ and analyzed by direct potentiometry with electrodes II and VI (Table 3), containing MIP material as active ingredient and lipophilic additive.

The practical CRT concentrations of these spiked samples obtained by ISE II were 137.9 and $295.8 \mu \mathrm{g} / \mathrm{mL}$ respectively. The corresponding relative errors were +2.0 and $-4.6 \%$, thereby suggesting that this determination was accurate. The corresponding standard deviations were 1.8 and $1.6 \%$, ascertaining the precision of the determination. Similar results were obtained by ISE VI. Average and standard deviation values of 124.9 and $279.9 \mu \mathrm{g} / \mathrm{mL}$ were obtained, respectively. These results corresponded to relative errors of -7.6 to $-9.7 \%$, respectively and standard deviations of 2.3 and $1.7 \%$, confirming again the accuracy and the precision of the potentiometric method. The average values of limit of detection, lower limit linear range and repeatability for ISEs II and VI were $9.0 \times 10^{-5} \mathrm{~mol} / \mathrm{L}, 7.73 \times 10^{-5} \mathrm{~mol} / \mathrm{L}$ and $2.0 \%$; and $7.7 \times$ $10^{-5} \mathrm{~mol} / \mathrm{L}, 7.1 \times 10^{-5} \mathrm{~mol} / \mathrm{L}$ and $2.3 \%$, respectively. Overall, the proposed method seemed suitable for CRT quantification in urine samples.

\section{Conclusions}

The molecular imprinting technique was applied successfully to produce polymeric materials acting as CRT host-tailored sensors in potentiometric transduction and plasticized PVC environment. The MIP materials gave rise to ISEs of good potentiometric analytical features when added of a lipophilic additive, capable of discriminating other CRT in aqueous media. These sensors displayed good selectivity and offered advantages such as simplicity in designing, short measurement time, good precision and high accuracy.

\section{References}

[1] WHO, World health Organization, Regional Office for Europe, http:// wwweurowhoint/en/what-we-do/health-topics/diseases-and-conditions/cancer (Accessed 2011).

[2] European Commission, Public Health, http://eceuropaeu/health/ph_determinants/ genetics/documents/cancer_screeningpdf assessed on the 9th of July of 2011.

[3] A. Steiber, J. Kerner, C.L. Hoppel, Carnitine: a nutritional, biosynthetic, and functional perspective, Mol. Asp. Med. 25 (5-6) (2004) 455-473.

[4] M.Y. Fong, J. McDunn, S.S. Kakar, Identification of metabolites in the normal ovary and their transformation in primary and metastatic ovarian cancer, PLoS One 6 (5) (2011) 1-12.

[5] T. Chen, G. Xie, X. Wang, J. Fan, Y. Qiu, X. Zheng, X. Qi, Y. Cao, M. Su, X. Wang, et al., Serum and urine metabolite profiling reveals potential biomarkers of human hepatocellular carcinoma, Mol. Cell. Proteomics 10 (7) (2011) 1-13.

[6] L.A. Calò, E. Pagnin, P.A. Davis, A. Semplicini, R. Nicolai, M. Calvani, A.C. Pessina AC, Antioxidant effect of L-carnitine and its short chain esters: relevance for the protection from oxidative stress related cardiovascular damage, Int J. Cardiol. 107 (2006) 54-56.

[7] A. Dayanandan, P. Kumar, C. Panneerselvam, Protective role of L-carnitine on liver and heart lipid peroxidation in atherosclerotic rats, J. Nutr. Biochem. 12 (5) (2001) 254-257.

[8] R.J. Thiel, S.W. Fowkes, Down syndrome and epilepsy: a nutritional connection? Med. Hypotheses 62 (1) (2004) 35-44.

[9] S. Savitha, J. Tamilselvan, M. Anusuyadevi, C. Panneerselvam, Oxidative stress on mitochondrial antioxidant defense system in the aging process: role of DL-alpha-lipoic acid and L-carnitine, Clin. Chim. Acta 355 (1-2) (2005) 173-180.
[10] S. Moretti, E. Alesse, L. Di Marzio, F. Zazzeroni, B. Ruggeri, S. Marcellini, G. Famularo S.M. Steinberg, A. Boschini, M.G. Cifone, et al., Effect of L-carnitine on human immunodeficiency virus-1 infection-associated apoptosis: a pilot study, Blood 91 (10) (1998) 3817-3824.

[11] U. Wenzel, A. Nickel, H. Daniel, Increased carnitine-dependent fatty acid uptake into mitochondria of human colon cancer cells induces apoptosis, J. Nutr. 135 (6) (2005) 1510-1514.

[12] B.J. Chang, M. Nishikawa, E. Sato, K. Utsumi, M. Inoue, L-Carnitine inhibits cisplatininduced injury of the kidney and small intestine, Arch. Biochem. Biophys. 405 (1) (2002) 55-64.

[13] K. Carvajal, R. Moreno-Sanchez, Heart metabolic disturbances in cardiovascular diseases, Arch. Med. Res. 34 (2) (2003) 89-99.

[14] A. Giancaterini, A. De Gaetano, G. Mingrone, D. Gniuli, E. Liverani, E. Capristo, A.V. Greco, Acetyl-L-carnitine infusion increases glucose disposal in type 2 diabetic patients, Metab. Clin. Exp. 49 (6) (2000) 704-708

[15] G. Mingrone, Carnitine in type 2 diabetes, Carnitine: the Science Behind a Conditionally Essential Nutrient 1033 (2004) 99-107.

[16] G.X. He, T. Dahl, Improved high-performance liquid chromatographic method for analysis of L-carnitine in pharmaceutical formulations, J. Pharm. Biomed. Anal. 23 (2-3) (2000) 315-321.

[17] I. Ferreira, M.N. Macedo, M.A. Ferreira, Enzymic flow injection determination of free L-carnitine in infant formulae, Analyst 122 (12) (1997) 1539-1541.

[18] A. Marzo, G. Cardace, N. Monti, S. Muck, E.A. Martelli, Chromatographic and nonchromatographic assay of L-carnitine family components, J. Chromatogr. Biomed. Appl. 527 (2) (1990) 247-258.

[19] A. Marzo, N. Monti, M. Ripamonti, E.A. Martelli, Application of high-performance liquid-chromatography to the analysis of propionyl-L-carnitine by a stereospecific enzyme assay, J. Chromatogr. 459 (1988) 313-317.

[20] Y.Z. Deng, J. Henion, J.J. Li, P. Thibault, C. Wang, D.J. Harrison, Chip-based capillary electrophoresis/mass spectrometry determination of carnitines in human urine, Anal. Chem. 73 (3) (2001) 639-646.

[21] Y.Z. Deng, N.W. Zhang, J. Henion, Chip-based quantitative capillary electrophoresis/ mass spectrometry determination of drugs in human plasma, Anal. Chem. 73 (7) (2001) 1432-1439.

[22] L. Vernez, W. Thormann, S. Krahenbuhl, Analysis of carnitine and acylcarnitines in urine by capillary electrophoresis, J. Chromatogr. A 895 (1-2) (2000) 309-316.

[23] R.P. Hassett, E.L. Crockett, Endpoint fluorometric assays for determining activities of carnitine palmitoyltransferase and citrate synthase, Anal. Biochem. 287 (1) (2000) 176-179.

[24] A. Galan, A. Padros, M. Arambarri, S. Martin, Automation of a spectrophotometric method for measuring L-carnitine in human blood serum, J. Autom. Chem. 20 (1) (1998) 23-26.

[25] S.H. Yalkowsk, G. Zografi, Potentiometric titration of monomeric and micellar acylcarnitines, J. Pharm. Sci. 59 (6) (1970) 798.

[26] C. Botre, F. Botre, C. Pranzoni, A potentiometric approach to the study of the antagonism between acetazolamide and L-carnitine congeners on carbonic-anhydrase activity, Electroanalysis 3 (6) (1991) 567-572.

[27] R.I. Stefan, J.F. van Staden, C. Bala, H.Y. Aboul-Enein, On-line assay of the Senantiomers of enalapril, ramipril and pentopril using a sequential injection analysis/amperometric biosensor system, J. Pharm. Biomed. Anal. 36 (4) (2004) 889-892.

[28] J. Zeravik, C. Teller, F.W. Scheller, Analysis of cholinesterase binding to a carnitinemodified EQCM sensor, Biosens. Bioelectron. 22 (9-10) (2007) 2244-2249.

[29] R.L. Stefan, R.G. Bokretsion, J.F. van Staden, H.Y. Aboul-Enein, Determination of Land D-enantiomers of carnitine using amperometric biosensors, Anal. Lett. 36 (6) (2003) 1091-1102.

[30] V.V. Cosofret, R.P. Buck, Recent advances in pharmaceutical analysis with potentiometric membrane sensors, Crit. Rev. Anal. Chem. 24 (1) (1993) 1-58.

[31] E. Bakker, E. Malinowska, R.D. Schiller, M.E. Meyerhoff, Anion-selective membrane electrodes based on metalloporphyrins-the influence of lipophilic anionic and cationic sites on potentiometric selectivity, Talanta 41 (6) (1994) 881-890.

[32] A. Kisiel, A. Michalska, K. Maksymiuk, Plastic reference electrodes and plastic potentiometric cells with dispersion cast poly(3,4-ethylenedioxythiophene) and poly(vinyl chloride) based membranes, Bioelectrochemistry 71 (1) (2007) 75-80.

[33] G.J. Guan, B.H. Liu, Z.Y. Wang, Z.P. Zhang, Imprinting of molecular recognition sites on nanostructures and its applications in chemosensors, Sensors 8 (12) (2008) 8291-8320.

[34] E. Busi, R. Basosi, F. Ponticelli, M. Olivucci, An innovative approach to the design of plastic antibodies: molecular imprinting via a non-polar transition state analogue, J. Mol. Catal. A Chem. 217 (1-2) (2004) 31-36.

[35] K. Hosoya, Y. Shirasu, K. Kimata, N. Tanaka, Molecularly imprinted chiral stationary phase prepared with racemic template, Anal. Chem. 70 (5) (1998) 943-945.

[36] B. Sellergren, Imprinted dispersion polymers-a new class of easily accessible affinity stationary phases, J. Chromatogr. A 673 (1) (1994) 133-141. 
[37] N. Lavignac, C.J. Allender, K.R. Brain, Current status of molecularly imprinted polymers as alternatives to antibodies in sorbent assays, Anal. Chim. Acta. 510 (2) (2004) 139-145.

[38] L. Ye, K. Mosbach, Molecularly imprinted microspheres as antibody binding mimics, React. Funct. Polym. 48 (1-3) (2001) 149-157.

[39] P. Manesiotis, A.J. Hall, J. Courtois, K. Irgum, B. Sellergren, An artificial riboflavin receptor prepared by a template analogue imprinting strategy, Angew. Chem. Int. Ed. 44 (25) (2005) 3902-3906

[40] M.C. Blanco-Lopez, M.J. Lobo-Castanon, A.J. Miranda-Ordieres, P. Tunon-Blanco, Electrochemical sensors based on molecularly imprinted polymers, TrAC Trends Anal. Chem. 23 (1) (2004)

[41] D. Kriz, O. Ramstrom, K. Mosbach, Molecular imprinting-new possibilities for sensor technology, Anal. Chem. 69 (11) (1997) A345-A349.

[42] A.C.B. Dias, E.C. Figueiredo, V. Grassi, E.A.G. Zagatto, M.A.Z. Arruda, Molecularly imprinted polymer as a solid phase extractor in flow analysis, Talanta 76 (5) (2008) 988-996

[43] F.X. Qiao, H.W. Sun, H.Y. Yan, K.H. Row, Molecularly imprinted polymers for solid phase extraction, Chromatographia 64 (2006) 625-634

[44] F.T.C. Moreira, V.A.P. Freitas, M.G.F. Sales, Biomimetic norfloxacin sensors made of molecularly-imprinted materials for potentiometric transduction, Microchim. Acta 172 (1) (2011) 15-23.
[45] F.T.C. Moreira, M.G.F. Sales, Biomimetic sensors of molecularly-imprinted polymers for chlorpromazine determination, Mater. Sci. Eng. C Mater. Biol. Appl. 31 (5) (2011) 1121-1128.

[46] A.H. Kamel, S.A.A. Almeida, M.G.F. Sales, F.T.C. Moreira, Sulfadiazine-potentiometric sensors for flow and batch determinations of sulfadiazine in drugs and biological fluids, Anal. Sci. 25 (3) (2009) 365-371.

[47] Y.M. Mi, E. Bakker, Determination of complex formation constants of lipophilic neutral ionophores in solvent polymeric membranes with segmented sandwich membranes, Anal. Chem. 71 (23) (1999) 5279-5287.

[48] H.Y. Yan, F.X. Qiao, K.H. Row, Molecularly imprinted-matrix solid-phase dispersion for selective extraction of five fluoroquinolones in eggs and tissue, Anal. Chem. 79 (21) (2007) 8242-8248.

[49] R.P. Buck, E. Lindner, Recommendations for nomenclature of ion-selective electrodes-(IUPAC Recommendations 1994), Pure Appl. Chem. 66 (12) (1994) 2527-2536.

[50] Y. Umezawa, P. Buhlmann, K. Umezawa, K. Tohda, S. Amemiya, Potentiometric selectivity coefficients of ion-selective electrodes. Part I. Inorganic cations-(technical report), Pure Appl. Chem. 72 (10) (2000) 1851-2082. 